\title{
Management of Anaphylaxis During the SARS-CoV-2 Pandemic
}

Kanwaljit K. Brar, $M D^{1}$

Albana Harizaj, $M D^{2}$ Anna Nowak-Wegrzyn, MD, PhD ${ }^{1,3, *}$

\author{
Address \\ *,1Department of Pediatrics, NYU Grossman School of Medicine, Hassenfeld Chil- \\ dren's Hospital, 160 East 32nd Street, LM3, New York, NY, 10016, USA \\ Email: Anna.nowak-wegrzyn@nyulangone.org \\ ${ }^{2}$ Department of Internal Medicine, Capital Health Medical Center, Pennington, NJ, USA \\ ${ }^{3}$ Department of Pediatrics, Gastroenterology and Nutrition, Collegium Medicum, \\ University of Warmia and Mazury, Olsztyn, Poland
}

Published online: 10 March 2021

(C) The Author(s), under exclusive licence to Springer Nature Switzerland AG part of Springer Nature 2021

\section{Supplementary Information}

This article is part of the Topical Collection on Anaphylaxis

The online version contains supplementary material available at https://doi.org/10.1007/s40521-021-00284-0.

Keywords Anaphylaxis; COVID-19 · SARS-CoV-2 - Emergency department · Epinephrine · Food allergy

\begin{abstract}
Purpose of Review Management of anaphylaxis during the SARS-CoV-2 pandemic should consider local infection rates so as to not burden local ED at times of pandemic, while also protecting patients from infection risks and progression of anaphylaxis. In this review, we identify a treatment strategy for anaphylaxis that balances the risks versus benefits of ED versus home management in this unprecedented time.

Recent Findings Physicians and patients have had to adapt new approaches to medical care during the SARS-CoV-2 pandemic due to restricted access to health care facilities. Telemedicine has substituted in-person visits, and such a drastic change in the patient care paradigm presents a need to revise the acute management of anaphylaxis.

Summary Physicians should utilize telemedicine during this time to engage in shared decision-making with patients and their families to devise an anaphylaxis plan of management that emphasizes home care when symptoms are mild with an exception for ED care if a patient has had severe, near-fatal anaphylaxis episodes in the past. Previous anaphylaxis recommendations should remain in place despite the pandemic, including prompt use of epinephrine when needed, avoidance of known allergens, training of patients and their caregivers, and carrying of epinephrine autoinjector devices at all times to remain prepared in the event of an anaphylaxis episode.
\end{abstract}




\section{Introduction}

Anaphylaxis is a severe "systemic hypersensitivity reaction," that is an acute, rapidly progressive, and lifethreatening event triggered by different allergens [1]. It is generally caused by an injection or ingestion of an allergen, with symptom manifestation that can vary in time and severity among individuals [1]. Anaphylaxis is an allergic emergency because it can cause shock and death. The current standard of care emphasizes prompt recognition, early administration of intramuscular epinephrine injection, and immediate activation of emergency services, followed by evaluation, further treatment, and observation under medical care. The unprecedented circumstances of the global 2020 SARS-Co$\mathrm{V}-2$ pandemic necessitated the re-evaluation of this management paradigm due to limited and delayed access to emergency services, long wait times in the crowded emergency departments (EDs), and the tangible risk of acquiring SARS-Co-V-2. In this review, we focus on these unexpected challenges and review the available evidence regarding the management of anaphylaxis during restricted access in the global 2020 SARS-Co-V-2 pandemic.

\section{Pathophysiology}

\section{Epidemiology}

Anaphylactic reactions can cause severe respiratory distress due to involvement of upper or lower airways, sometimes simultaneously. In fatal cases, anaphylaxis manifests with laryngeal edema, bronchial obstruction, and hyperinflation of the lungs $[2 \bullet \bullet]$. Microscopic presentation of anaphylaxis in the bronchi are luminal secretions, peribronchial congestion, submu-

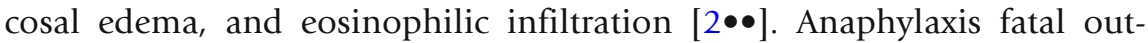
comes can be caused by hypoxia and/or vascular collapse depending on the allergen. On physical exam, flushing, diffuse erythema, giant hives,

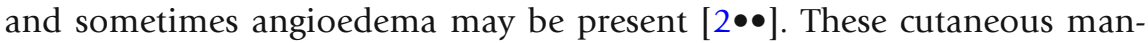
ifestations are attributed to the release of endogenous histamine. The acute phase anaphylaxis reaction involves mast cells and basophils through the mechanism of IgE binding and cross-linking of the highaffinity IgE receptors on their surfaces. Platelets, neutrophils, macrophages, and monocytes could also be present in anaphylaxis reactions, particularly in late-phase or biphasic anaphylaxis. These involve different signaling events through mediators, such as complement mediators, cysteinyl leukotrienes, histamine, IL-1, IL-6, TNF $\alpha$-receptor 1, plateletactivating factor, and serum tryptase, which may be amplified during an anaphylactic reaction $[2 \bullet \bullet, 3 \bullet]$.

The prevalence of anaphylaxis is increasing in developed countries; in the USA, it is estimated to be at least $1.6 \%$ among the general adult population as shown in a random-digit-dial survey of adults from 2003 to 2013, and 1 in 50 Americans adults may experience anaphylaxis $[3 \bullet, 4]$. Triggers for anaphylaxis can vary between adults and kids; in adults, medications are most common (34\%), followed by foods (31\%), and then insect stings (20\%), whereas in children, foods are most commonly implicated [4]. In the pediatric population, the prevalence of food-induced anaphylaxis increased $50 \%$ in the timeframe 
2007-2012 likely because the prevalence of reported food allergy among US children increased $18 \%$ over 10 years from 1997 to 2007 [5••, 6, 7]. The lifetime prevalence of anaphylaxis has been estimated at $1.6 \%$ to $5.1 \%$ [8].

ED visits have increased by $101 \%$ in the timeframe of 2005-2014, with the pediatric population and adults greater than 65 years old having the predominance of visits [9]. The first line of treatment for uniphasic or biphasic anaphylaxis reactions is epinephrine, and administration must be prompt within minutes of onset of symptoms. However, most studies indicate that epinephrine is under-utilized, both in the home and ED setting [4]. Antihistamines and glucocorticoids are second-line treatments and may only have benefit in instances of cutaneous or otherwise mild reactions involving a single system only. Depending on the severity or duration of a reaction, a second dose of epinephrine might be indicated. Patients should be monitored in ER for 4-6 h before

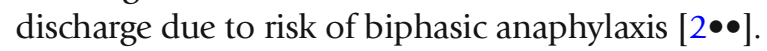

Fatal Anaphylaxis

Death from anaphylaxis is a rare event. In one epidemiologic study using 3 national databases, case fatality rates of anaphylaxis in the USA were between 0.25 and $0.33 \%$ of all anaphylaxis cases presented to a hospital or Emergency Department (ED) with $77 \%$ of fatality outcomes occurring in the hospitalized patients [10]. In the largest study of pediatric anaphylaxis cases in the USA and Canada from 2010 to 2015, 1\% of the pediatric population had a fatal outcome from anaphylaxis, with food being the most common cause of these events

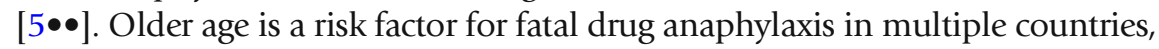
including the UK and Australia, which may be due to increased cardiovascular

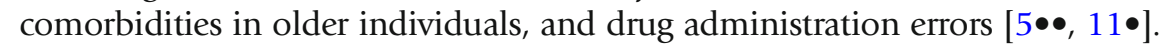
In the USA, African-American race may also be a risk factor for fatal drug anaphylaxis $[11 \bullet]$.

\title{
Unique Challenges to the Management of Anaphylaxis During COVID-19
}

\author{
Diagnosis of Anaphylaxis
}

Diagnosis of anaphylaxis is based on recognition of the clinical constellation of symptoms because no rapid screening test is available. During the present pandemic, ED physicians frequently are faced with the dilemma of the correct diagnosis of anaphylaxis versus SARS-CoV-2 illness. Both conditions are characterized by severe systemic reaction that triggers a cytokine release storm. These medical events, when the patient is presented to the ED, may be very similar. If the onset event history is not available or not revealing, laboratory tests such as allergen-specific IgE and serum tryptase drawn optimally within 15-180 min (up to $18 \mathrm{~h}$ ) after symptom onset can help the physician to differentiate between SARS-CoV-2 and anaphylactic shock [12]. In comparison, SARS-CoV2 cases show a wide range of clinical presentations starting from a mild, selflimiting viral respiratory disease to extensive pneumonia, endothelitis, thromboembolic events, and multiorgan failure as the most severe presentation. Laboratory results in COVID-19-infected patients showed severe lymphopenia and eosinopenia $[13 \bullet]$. Anaphylactic reactions can progress in severity if the 
patient also has an ongoing infection. When the global society is suddenly faced with a global pandemic, it becomes crucial for a physician to correctly and promptly differentiate and diagnose anaphylactic shock and very similar conditions caused by non-allergic factors.

Based on the October 2020 World Health Organization (WHO) report, COVID-19 cases have exceeded more than 30 million worldwide, and over 7 million cases in the United States (US) with death events exceeding 1 million and 200,000 , respectively $[13 \bullet, 14]$. The case fatality rate is approximately $3 \%$ in the USA, or 56 deaths per 100,000 people [13•]. Center for Disease Control and Prevention (CDC) data released in September 2020 show that in the USA, the age distribution of COVID-19 deaths is less than $0.1 \%$ for children $0-17$ years, $0.5 \%$ for adults $18-29$ years, $1.3 \%$ for $30-39$ years, $3.2 \%$ for $40-$ 49 years, and then jumping to $15.6 \%$ for $50-64$ years, $21.1 \%$ for $65-74$ years, $26.4 \%$ for $75-84$ years, and $31.7 \%$ for adults above 85 years old, making advanced age the biggest risk for mortality for COVID-19 just as it is for anaphylaxis $[12,14,15]$.

Case fatality rate calculated based on CDC data on age distribution is $0.04 \%$ among children $0-4$ years, $0.018 \%$ among children age $5-17,0.8 \%$ in adults age $18-64$, and $15.8 \%$ in adults above 65 years old.

\section{Delayed Access to Emergency Care}

The high COVID-19 infection rate among the general population and overflow of hospitals with COVID-19 patients during peak time of COVID-19 pandemic had a negative side effect on patients with other life-threatening conditions such as anaphylaxis, as it resulted in patients hesitating to seek or delaying emergent medical care.

An academic allergy unit in Milan, Italy, which was previously an epicenter of COVID-19 infection, proposed measures to navigate the epidemic $[16 \bullet \bullet]$. These included social distancing measures in their clinic, and checkpoint measures for screening patients, which have now become

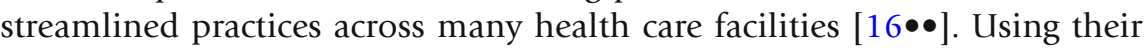
guidance, in-person appointments would be restricted for high-risk poorlycontrolled patients that require more careful follow-up. These patients, such as those with severe asthma, are at increased risk for severe anaphylaxis and should have continuity of care in the ambulatory setting despite

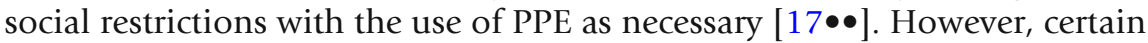
visits, which place patients at risk for anaphylaxis in the clinic setting, should be restricted dependent upon local transmission risk, such as buildup dosing of immunotherapy, spirometry, nebulized treatments, or diag-

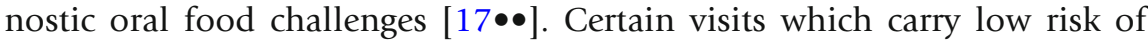
anaphylaxis can be transitioned to home, such as administration of biologics with the exception of omalizumab $[17 \bullet \bullet, 18]$. The emphasis should be on continuation of medical care so as to not have patient's medical conditions worsen in these unprecedented times, including the continuation of daily controller medicine for asthma $[16 \bullet \bullet, 18]$.

\section{Revised Anaphylaxis Management Algorithm}

The current change in the healthcare landscape contributed to a revised anaphylaxis management algorithm published in June 2020 when the COVID-19 
pandemic severely impacted access to care in New York City [19••]. In this approach, management of anaphylaxis with epinephrine can be considered at home, unless the patient has had severe, near-fatal anaphylaxis episodes in the past (see Fig. 1). The algorithm highlights the use of telemedicine to establish good communication with families for a plan for access to care in case of an

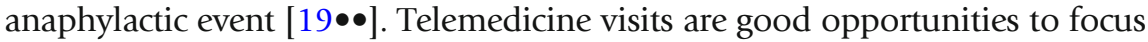
on education for families including avoidance of triggers, reasons to administer epinephrine, and careful assessment for which events should be evaluated in the ED, particularly for those patients at risk for severe anaphylaxis, such as those with asthma. Telemedicine visits are also opportunities to provide reassurance during this stressful time and empower patients for home management if necessary $[17 \bullet \bullet, 18]$.

Families should be advised of signs and symptoms of biphasic and protracted anaphylaxis and reasons for second epinephrine administration. They should also be advised of COVID-19 specific concerns, such as the risk for virus aerosolization with the use of nebulizers in case someone with anaphylaxis also happens to have active coronavirus infection $[17 \bullet \bullet]$. They can be advised of the use of 2-6 puffs of Albuterol if under 4 years, or $4-10$ puffs if over 4 years as an alternative treatment in place of nebulized bronchodilator in case of wheezing or other respiratory symptoms with an anaphylactic event [20•]. They should also be advised to check that devices are unexpired and in good condition. For patients with concomitant asthma, they should make sure inhaler devices have enough metered dose medication and are also unexpired.

Thus, all patients at risk for anaphylaxis should carry at least two unexpired epinephrine auto-injectors and have them available at all times, along with a personalized action plan with guidelines for administration $[1,17 \bullet \bullet, 18]$. In contrast to the recommendations of most anaphylaxis plans, which emphasize calling 911 after epinephrine use, patients can be advised to make decisions based upon symptom response to epinephrine and as guided by their allergist $[21 \bullet \bullet]$. Preventive anaphylaxis care, such as a telemedicine allergist's visit, and preemptive prescription fill for epinephrine have been shown to lower the risk for anaphylaxis severity as patient's may better recognize their symptoms and use epinephrine promptly [22].

\section{Management of Food Allergy During SARS-CoV-2 Pandemic}

We pay special attention to the management of food allergy because of the importance of foods as triggers of anaphylaxis in the outpatient setting, across all age groups. Many of the previous recommendations for food allergy management, particularly in schools remain unchanged despite the pandemic, with an emphasis on allergen avoidance, use of anaphylaxis plans, and availability of and training on use of epinephrine autoinjector devices. The CDC released guidelines on in-person learning during the pandemic, which emphasized cleaning surfaces, handwashing, and social distancing measures in the classroom, and during lunchtime, including the recommendation that children eat lunch in the

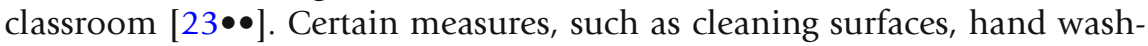
ing, and avoidance of sharing food, will have a benefit of reducing food 


\title{
Revised anaphylaxis management algorithm during \\ SARS COV-2 pandemic \\ to be implemented based on the local risk / benefit assessment
}

\begin{abstract}
A Patients with history of severe anaphylaxis such as those who have been intubated and ventilated, or had reactions treated with more than 2 doses of epinephrine should follow their routine anaphylaxis plan and activate emergency services immediately when anaphylaxis is recognized.

IMPORTANT REMINDER: Anaphylaxis is a potentially life-threatening, severe allergic reaction.

If in doubt, give epinephrine.

\section{SEVERE SYMPTOMS: any of the following}

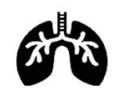

LUNG

Shortness of breath Wheezing, repetitive cough

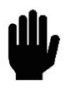

SKIN

Many hives over body, widespread redness

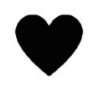

HEART

Pale or bluish skin, faintness, weak pulse, dizziness

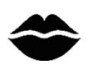

MOUTH

Significant swelling of the tongue or lips

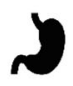

GUT

Repetitive vomiting, severe diarrhea

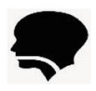

THROAT

Tight or hoarse throat, trouble breathing or swallowing

Mild symptoms from more than one system area:

Itchy runny nose, sneezing and/or

Itchy mouth and/or

Few hives, mild itch

and /or

Mild nausea or discomfort

\section{INJECT EPINEPHRINE IMMEDIATELY}

while seated and have telephone within reach

2. Notify a housemate or neighbor to help you

3. Lay down with legs elevated near the doorway which should be unlocked or open to allow others to enter and help. Keep children in apposition of comfort, to minimize respiratory distress and agitation and risk of aspiration in case of emesis
\end{abstract}

4. Administer oral antihistamine, preferably non-sedating cetirizine

5. Administer albuterol for respiratory symptoms if prescribed and available

6. Monitor symptoms and blood pressure/pulse if possible

SYMPTOMS DON'T IMPROVE OR WORSEN

SEVERE SYMPTOMS RESOLVE

Repeat epinephrine injection in $\mathbf{5}$ min or sooner if symptoms escalate rapidly

SEVERE SYMPTOMS

DON'T IMPROVE OR WORSEN

SEVERE SYMPTOMS RESOLVE

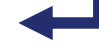

Cont

Be ready to administer treatment if symptoms reappear (biphasic anaphylaxis)

Notify your physician on a non-urgent basis

Replenish emergency medications

\section{Activate emergency services (Call 911)}

Fig. 1. A revised management algorithm for use during SARS CoV-2 pandemic in place of a standard management protocol 


\section{Conclusions}

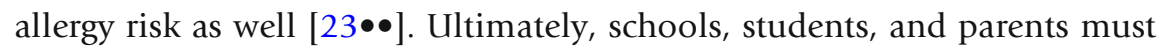
engage in shared decision-making to determine the best course of action for the prevention of anaphylaxis in schools during this unprecedented time $[23 \bullet \bullet]$.

The pandemic may be presenting itself as an opportunity for allergy medicine to evolve with the use of telemedicine visits, and increased comfort around

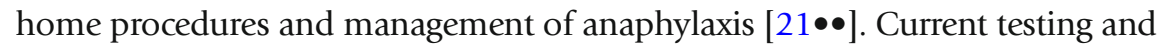
visit limitations will result in less screening for food allergy in high risk children, such as infants with eczema and egg allergy, and subsequently less supervised food challenges. This may result in a more cost-effective health care model and fewer unnecessary medical visits with a shifted focus on home introductions and home challenges $[21 \bullet \bullet]$. Home introductions are generally safe, with reaction rates of approximately $2 \%$ that are typically cutaneous. These introductions or challenges could be augmented with a virtual visit, and providers can consider pre-emptive epinephrine autoinjector prescription in case of reac-

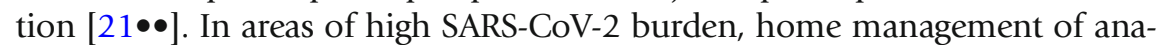
phylaxis can be considered if families are willing and have access to two epinephrine autoinjectors as advised, and there is no previous history of mul-

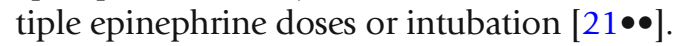

In conclusion, decisions regarding the management of anaphylaxis during the SARS-CoV-2 pandemic should consider local infection rates and the risk versus benefits of ED versus home management so as to not burden local ED at times of pandemic, while also protecting patients from infection risks and progression of anaphylaxis. Continuation of medical care during the pandemic, education of patients and families with regard to the importance of strict allergen avoidance, recognition of symptoms and severity, and prompt epinephrine use will help facilitate appropriate home management in areas of high SARS-CoV-2 burden. Adherence to current guidelines about management of uniphasic and biphasic anaphylaxis is very important to avoid fatal outcomes.

\section{Supplementary Information}

The online version contains supplementary material available at https://doi.org/10.1007/s40521-021-00284-0.

\section{Data Availability}

Not applicable

\section{Code Availability}

Not applicable 


\section{Author Contribution}

All authors contributed to reviewing and analyzing the relevant literature and drafting the manuscript.FundingDepartment of Pediatrics, NYU Grossman School of Medicine, Hassenfeld Children's Hospital, New York, NY, USA

\section{Declaration}

\section{Conflict of Interest}

Anna Nowak-Wegrzyn received research support from National Institute of Allergy and Infectious Diseases, DBV Technologies, Astellas Pharma, Danone, and Nestle and consultancy fees from Regeneron and Gerber Institute; she serves as the Deputy Editor for the Annals of Allergy, Asthma and Immunology and the Chair of the Medical Advisory Board of the International FPIES Association.

Kanwaljit K. Brar and Albana Harizaj report no conflicts.

\section{References and Recommended Reading}

Papers of particular interest, published recently, have been highlighted as:

- Of importance

$\bullet \quad$ Of major importance

1. Simons FER, Ledit A, Bilo $\mathrm{MB}$, et al. International consensus on (ICON) anaphylaxis. World Allergy Org J. 2014;7:9.

2.• Shaker MS, Wallace DV, Golden DBK, et al. Anaphylaxis a 2020 practice parameter update, systematic review, and Grading of Recommendations, Assessment, Development and Evaluation (GRADE) analysis. J Allergy Clin Immunol. 2020;145:1082-12.

The important paper providing the latest critical updates in anaphylaxis.

3.• Reber LL, Hernandez JD, Galli SJ, et al. The pathophysiology of anaphylaxis. J Allergy Clin Immunol. 2017;140:335-4.

Very good general overview of pathophysiology of anaphylaxis.

4. Wood RA, Camargo CA, Lieberman P, et al. Anaphylaxis in America: the prevalence and characteristics of anaphylaxis in the United States. J Allergy ClinImmunol. 2014;133:461-7.

5.• Ramsey NB, Guffey D, et al. Epidemiology of anaphylaxis in critically ill children in the United States and Canada. J Allergy Clin Immunol Pract. 2019;7:224.

Excellent paper analyzing recent data in the epidemiology of anaphylaxis.

6. Branum AM, Lukacs SL. Food allergy among children in the United States. Pediatrics. 2009;124:1549-55.
7. Parlaman JP, Oron AP, Uspal NG, DeJong KN, Tieder JS. Emergency and hospital care for food-related anaphylaxis in children. Hosp Pediatr. 2016;6:269-74.

8. Alvarez-Perea A, Ameiro B, Morales C, Zambrano G, Rodríguez A, Guzmán $M$, et al. Anaphylaxis in the pediatric emergency department: analysis of 133 cases after an allergy workup. J Allergy Clin Immunol Pract. 2017;5:1256-63.

9. Motosue MS, Bellolio MF, Van Houten HK, et al. Increasing emergency department visits for anaphylaxis, 2005-2014. J Allergy Clin Immunol Pract. 2017;171175.

10. Borish L, Danoff T, Ma L. Death from anaphylaxis is a reassuringly unusual outcome. J Allergy Clin Immunol. 2013;133(4):AB234.

11. Turner PJ, Jerschow E, Umasunthar T, et al. Fatal anaphylaxis: mortality rate and risk factors. J Allergy Clin Immunol Pract. 2017;5:1169-7.

This paper provides an important insight into fatal anaphylaxis.

12. Simons FER, Ebisawa M, Sanchez-Borges M, Thong BY, Worm M, Tanno LK, et al. 2015 update of the evidence base: World Allergy Organization anaphylaxis guidelines. World Allerg Org J. 2015;8:32.

13. Alvarez-Perea A, Baeza ML. Anaphylactic shock following the diagnosis of coronavirus disease 2019. Ann Allergy Asthma Immunol. 2020;125(4):477-. 
Very interesting perspective of differentiating between anaphylactic shock and COVID-19 patient presentation.

14. World Health Organization. WHO Coronavirus Disease (COVID-19) Situation Dashboard In: WHO Homepage. World Health Organization. 2020. https:// covid19.who.int/. Accessed 28 Aug 2020.

15. Center for Disease Control and Prevention. Demographic trends of COVID-19 cases and deaths in the US reported to CDC. In: Deaths by Age Group chart. Center for Disease Control and Prevention. 2020. https:// covid.cdc.gov/covid-data-tracker/\#demographics. Accessed 20 Sept 2020.

16.• Malpiero F, Paoletti G, Puggioni F, et al. An academic allergy unit during COVID-19 pandemic in Italy. J Allergy Clin Immunol. 2020;146(1):22.

Excellent paper on allergic patient management and treatment adaptation during pandemic in Italy.

17.• Shaker MS, Oppenheimer J. Grayson. COVID-19: pandemic contingency planning for the allergy and immunology clinic. J Allergy Clin Immunol Pract. 2020;8(5):1477-1488.e.

Important article covering the measures that have to be taken for allergic patients during pandemic lockdown.

18. Codispoti CD, Bandi S, Moy J, Mahdavinia M. Running a virtual allergy division and training program in the time of COVID-19 pandemic. J Allergy Clin Immunol. 2020;145:1357-9.

19.• Casale TB, Wang J, Nowak-Wegrzyn A. Acute at home management of anaphylaxis during the COVID-19 pandemic. J Allergy Clin Immunol Pract. 2020;8(6):1795-.
Excellent and unique approach in advising both patients and medical doctors how to plan on managing acute anaphylaxis at home.

20. Levin M, Ansotegui IJ, Bernstein J, et al. Acute asthma management during SARS-CoV2-pandemic 2020. World Allergy Organ J. 2020;13(5):10012.

Informative paper in addressing asthma management.

21.• Mack DP, Chan ES, Shaker M, et al. Novel approaches to food allergy management during COVID-19 inspire long-term change. J Allergy Clin Immunol Pract. 2020. https://doi.org/10.1016/j.jaip.2020.07.020 This article describes management of patients experiencing food allergy during pandemic lockdown.

22. Clark S, Wenhui W, Rudders SA, et al. Risk factors for severe anaphylaxis in patients receiving anaphylaxis treatment in US emergency departments and hospitals. J Allergy Clin Immunol. 2014;134(5):1125-30.

23.• Greenhawt M, Shaker M, Stukus DR, et al. Managing Food Allergy in Schools During the COVID-19 Pandemic. J Allergy Clin Immunol Pract. 2020. https://doi. org/10.1016/j.jaip.2020.07.016 Great article on food allergy management in schools during pandemics.

\section{Publisher's Note}

Springer Nature remains neutral with regard to jurisdictional claims in published maps and institutional affiliations. 